\title{
An automated analogue of the one-way Miller-type avoidance box ${ }^{1,2}$
}

MORRIE BAUM AND SAMUEL A, BOBROW ${ }^{3}$

UNIVERSITY OF PENNSYLVANIA

A novel, fully automated avoidance apparatus analogous to the one-way Miller-type avoidance box was described, and the learning obtained with it was more rapid than typical shuttlebox avoidance acquisition. Reasons why rapid learning is obtained using both the one-way manual avoidance technique and its automated analogue, but not with the shuttlebox, were discussed.

Many investigators have noted the relative difficulty of training rats to avoid shock in both the shuttlebox and the lever-press situation, as compared to the rapid learning found with a one-way avoidance box of the type used by Miller (1948). A variety of techniques has been suggested to facilitate avoidance acquisition. Thus, D'Amato \& Fazzaro (1966) have advocated the use of a discontinuous, pulsing shock in the lever-press avoidance training of rats. Ray (1966) obtained rapid shuttlebox learning employing an air-blast UCS rather than

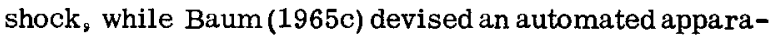
tus somewhat analogous to the jump box of Maatsch (1959) and obtained very rapid acquisition.

The hand-run, one-way box has several advantages over other types of avoidance apparatus. In addition to relatively rapid acquisition, one-way avoidance learning is sensitive to prior fear-conditioning manipulations (Baum, 1965a, b; Black, 1965), while shuttlebox learning, for example, is less so (Mullin \& Mogenson, 1963). Moreover, the one-way box has been one of the most frequently used avoidance apparatuses and has been improved in design. Working with cats, Lubar (1964) and McCleary (1961) used a modified one-way avoidance box having detachable compartments which could be manually disengaged and interchanged, thus permitting unidirectional avoidance training without the necessity of picking up and handling $S$ before each trial. Kirby (1963) utilized a somewhat similar apparatus with rats.

The present study describes a completely automated analogue of the one-way Miller box. In addition to the obvious advantages of automation, the apparatus described here provides a new tool for studying why oneway avoidance acquisition differs from shuttlebox learning, or, more generally put, why some avoidance tasks are easier than others.

Subjects

The Ss were eight male albino rats of the SpragueDawley strain obtained from the Holtzman Co., Madison, Wisconsin. They were maintained on ad lib food and water, and were housed two to a cage in a rat colony room for at least one week before training. At the time of training, the rats were 100-130 days old and weighed 300 to $350 \mathrm{gm}$.

\section{Apparatus and Procedure.}

Figure 1 is a sketch of the apparatus, which was essentially a shuttlebox mounted on a turntable driven by a reversible motor. The box was made of plywood and was 24 in. long $x 5$ in. wide $x 7$ in. high. The grid floor, through which shock could be delivered, was made of $1 / 8$ in. diameter copper bars set at intervals of $3 / 4$ in. along the bottom of the box. The shock itself was scrambled $110-\mathrm{v}$ ac delivered through a $100 \mathrm{~K}$ resistor in series with the rat. The box was divided into equalsized compartments by a swinging door hinged at the ceiling. The door was held in a fixed position by means of a solenoid device mounted on the side of the box, in order to prevent responding during the intertrial interval. Retraction of the solenoid permitted the rat to push through the swinging door and cross from one compartment to another. Both compartments were painted aluminum gray, while the door separating them had its upper half painted aluminum and the lower half painted black. The rats' presence in either compartment was detected by means of two photorelays mounted on the outside of the box.

The ceilings over the two compartments were transparent Plexiglas panels which permitted observation and which also served as "doors" to the apparatus, since they could be slid open and shut to permit putting $S$ into the apparatus at the start of the session, and removal after training was completed. As seen in Fig. 1,

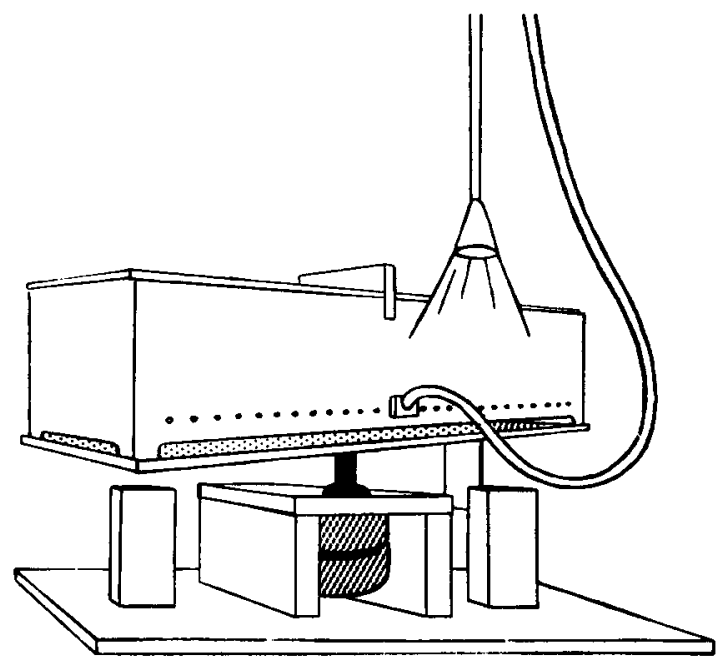

Fig. 1. Simplified sketch of the apparatus, showing the avoidance box sitting atop the motor driven tumtable, the fixed external light cue, and the cable leading from the avoidance box to the programming equipment. 
a light ( $7 \mathrm{w}$ ) was positioned over the apparatus to provide a fixed external cue. The side of the avoidance box under the light was always the safe side, i.e., the compartment to which S escaped or avoided, and the side in which $\mathrm{S}$ spent the shock-free intertrial interval. The motor-driven turntable was used to present the next trial by rotating the apparatus through 180 degrees, thus causing the side which was "safe" during the preceding intertrial interval to now become "dangerous," and vice versa. Figure 1 shows the motor in the process of rotating the apparatus to presenta new trial. A reversible motor was used since continued rotation in the same direction would cause the wires running from the apparatus to the nearby programming equipment and shock scrambler (see the cable in Fig. 1) to become fouled.

The avoidance procedure was completely automated. The CS for avoidance was compound, consisting of (a) feedback from being rotated at the start of each trial, (b) light offset as $\mathrm{S}$ was rotated from the position under the light to the "danger" position which was in relative darkness, (c) the click of the solenoid which freed the swinging door and permitted S's responding, and (d) onset of a loud, $78 \mathrm{db}$ buzzer situated near the apparatus (both (c) and (d) occurring simultaneously when the 180 degree rotation had been completed). The CS-UCS interval began immediately after rotation and was $10 \mathrm{sec}$., after which shock came on and remained on until $S$ escaped. $S$ could avoid by responding within $10 \mathrm{sec}$. of buzzer onset. When S responded (escaped or avoided), the buzzer immediately terminated, the solenoid released to prevent crossing back in the intertrial interval, and, of course, $S$ found itself in the "safe" position under the light, where it spent the next $45 \mathrm{sec}$. (the intertrial interval), after which the next trial was presented. Ss were trained to a criterion of 10 consecutive avoidances, or until a total of 50 trials had been presented.

\section{Results and Discussion}

Seven of the eight animals achieved the learning criterion within 50 trials. The group as a whole required a median of 6.0 trials to the first avoidance response, and a median of 11.0 trials and 7.5 shocks before achieving the learning criterion. Thus, avoidance learning in the novel, automated analogue of the one-way Miller box is considerably more rapid than shuttlebox acquisition and compares quite favorably with the handrun one-way situation (Baum, 1965c, reviews the data for these latter situations). Furthermore, $50 \%$ of the animals $(4 / 8)$ attained the criterial run of ten consecutive avoidance responses upon making the first avoidance response. This all-or-none aspect of avoidance learning has been thought by some to characterize a simple avoidance task (Theios \& Dunaway, 1964).
Apart from the advantages of automation, the apparatus and data presented above help to shed new light on why one-way avoidance is more easily learned than shuttlebox avoidance. Rotating the apparatus in this report is functionally equivalent to manually returning $S$ to the start box for the next trial. In both the manual procedure and the automated analogue described above, the feature of unidirectionality is conserved-i.e., to avoid successfully, $S$ has only to run in the same geographical direction on every trial. In contrast, shuttlebox learning lacks two features present in both the automated anaiogue and hand-run one-way procedures; (a) the feature of unidirectionality, and (b) the added cue of being rotated or handled before each trial. We are currently performing experiments to further elucidate what aspect of shuttlebox training causes the difficulty in learning.

\section{References}

Baum, M. The recovery-from-extinction of an avoidance response following an inescapable shock in the avoidance apparatus. Psychon. Sci., 1965a, 2, 7-8.

Baum, M. "Reversal learning" of an avoidance response as a function of prior fear conditioning and fear extinction. Canad. J. Psychol., 1965b, 19, 85-93.

Baum, M. An automated apparatus for the avoidance training of rats. Psychol. Rep., 1965c, 16, 1205-1211.

Black, A. H. In W. F. Prokasy (Ed.), Classical conditioning. New York: Appleton-Century-Crofts, 1965. Pp. 39-40.

D'Amato, M. R., \& Fazzaro, J. Discriminated lever-press avoidance learning as a function of type and intensity of shock. $J$. comp. physiol. Psychol., 1966, 61, 313-315.

Kirby, R. H. Acquisition, extinction, and retention of an avoidance response in rats as a function of age. J. comp. physiol. Psychol., $1963,56,152-162$

Lubar, J. F. Effect of medial cortical lesions on the avoidance behavior of the cat. J. comp. physiol. Psychol., 1964, 58, 38-46.

Maatsch, J. L. Learning and fixation after a single shock trial. J. comp. physiol. Psychol, 1959, 52, 408-410.

McCleary, R. A. Response specificity in the behavioral effects of limbic system lesions in the cat. J. comp. physiol. Psychol., $1961,54,605-613$.

Miller, N. E. Studies of fear as an acquirable drive: I. Fear as motivation and fear reduction as reinforcement in the learning of new responses. $J$. exp. Psychol., 1948, 38, 89-101.

Mullin, A. D., \& Mogenson, G. J. Effects of fear conditioning on avoidance learning. Psychol. Rep., 1963, 13, 707-710.

Ray, A. J., Jr. Shuttle avoidance: Rapid acquisition by rats to a pressurized air unconditioned stimulus. Psychon. Sci., 1966 , $5,29-30$.

Theios, J., \& Dunaway, J. E. One-way vs shuttle avoidance conditioning. Psychon. Sci., 1964, 1, 251-252.

\section{Notes}

1. This research was supported by USPHS Grant No. MH-04202 and NSF Grant No. GB-2428, both to Richard L. Solomon. The authors are grateful to Dr. Solomon for his encouragement and advice. 2. Reprints of this article and a more detailed description of the construction and programming of the apparatus described here are available from either author.

3. Now at Stanford University. 